\title{
IRAS observations of extended planetary nebulae
}

\author{
A. Leene and S.R. Pottasch \\ Kapteyn Astronomical Institute, \\ Groningen, \\ The Netherlands
}

The pointed observations made by the IRAS satellite have been analysed. In total 67 nebulae have been observed of which 10 proved to be resolved. The majority of the observations were carried out with the CPC and with the DSD macro of the survey array (Leene \& Pottasch, 1987b). Further special deep maps were made of six nebulae (Leene \& Pottasch, 1987a; Leene, 1987). Typical noise levels of the deep images are 0.03-0.1 $\mathrm{MJy} \mathrm{ster}^{-1}$.

The distribution of emission around the central star confirms that the 12 and $25 \mu \mathrm{m}$ emission are due to ionic line emission. This is very clear in the nebula NGC 7293 (Leene \& Pottasch, 1987a) and in NGC 6853 (Zhang et al., 1987). The other nebulae (A7, A21, A31, A35 and NGC 1360) confirm these findings. The $12 \mu \mathrm{m}$ emission is most likely due to the NeII $(12.81 \mu \mathrm{m})$ or due to the SIV $(10.52 \mu \mathrm{m})$ line. The $25 \mu \mathrm{m}$ band shows a strong central contribution due to the OIV $(25.87 \mu \mathrm{m})$ line and a halo of the SIII $(18.68 \mu \mathrm{m})$ line.

The sizes of the nebulae at 50 and $100 \mu \mathrm{m}$ of the resolved pointed observations are very similar, indicating a lack of a temperature gradient. The radial profiles of the other nebulae seem to confirm this. Thus the dust is uniformly heated, probably by trapped Lymann $\alpha$ photons. The infrared sizes compared to the optical sizes confirm this heating mechanism. In nearly all cases the infrared size is smaller than the $\mathrm{H} \alpha$ size. The only exception are the two "young" nebulae NGC 6543 and $\mathrm{BD}+30$ 3639. In these nebulae the infrared size is much larger than the $\mathrm{H} \alpha$ size.

The observations of A7, A31 and A35 show that infrared emission is still detectable for very low photographic surface brightness planetary nebulae (25-26 mag $\left.\operatorname{arcsec}^{-2}\right)$. This allows to make images of nearly all nebulae in the Abell catalogue. No evidence could be found for infrared emission beyond the optical image.

Only in the nebulae NGC 6543 and BD +303639 possible evidence for the remnant of an AGB wind can be found. This confirms the scenario of Barlow (1983) in which young nebulae still have an optical thick shell. This allows a coexistence of an AGB remnant and an ionized region. However when the shell expands it becomes optical thin and the whole AGB shell becomes a HII region.

The lack of dust seen in the 12 and $25 \mu \mathrm{m}$ band is somewhat in contrast with the observations of NGC 7027. In this nebulae there is a strong contribution of very small grains (PAH's). It is not unlikely that these small grains are destroyed by the UV radiation in the large nebulae. Only in the young nebulae with an optical thick shell the right environment is present to let these small grains survive. The lack of an infrared halo can easily be explained by the "remnant" of an AGB wind. The dust seen in planetary nebulae can be provided by an AGB wind if it reaches a maximum mass loss rate of $10^{-4} \mathrm{M}_{\odot} \mathrm{yr}^{-1}$. Such a mass loss rate is also observed in non-variable OH/IR stars, which are possible precursors of planetary nebulae (van der Veen et al., 1987).

\section{References.}

Barlow, M.J.: 1983, IAU Symp. 103, p105, Reidel

Leene, A., Pottasch, S.R.: 1987a, Astron. Astrophys. 173, 145

Leene, A., Pottasch, S.R.: 1987b, submitted to Astron. Astrophys.

Leene, A.: 1987, Ph.D. thesis, University Groningen, The Netherlands.

van der Veen, W., Habing, H.J., Geballe, T.: 1987, "Planetary and proto-planetary nebulae: from IRAS to ISO", p69, ed. A. Preite-Martinez, Reidel

Zhang, C.Y., Leene, A., Pottasch, S.R., Mo, J.E.: 1987, Astron. Astrophys. 178, 247 